\title{
Percutaneous coronary intervention of a circumflex artery chronic total occlusion using the retrograde approach via ipsilateral collateral circulation from the left anterior descending artery
}

\author{
Rodrigo Wainstein, Vladimír Džavík \\ Interventional Cardiology Program, Peter Munk Cardiac Centre, University Health Network, Toronto, Ontario, Canada
}

\section{ARTICLE INFO}

Article history:

Received: 23 May 2012

Accepted: 24 May 2012

Available online: 7 June 2012

\section{Keywords:}

Chronic total occlusion

Ipsilateral

Percutaneous coronary

intervention

Retrograde approach

\section{ABSTRACT}

Retrograde recanalization techniques have markedly improved success rates of attempts to open chronic coronary occlusions in symptomatic patients. A contralateral artery with a collateral channel to the distal segment of the occluded artery is traversed and the vessel is then wired and dilated retrogradely. Occasionally only an ipsilateral collateral is available. We describe the first case of retrograde recanalization of a dominant circumflex artery via a septal collateral channel and discuss the issues surrounding the use of the ipsilateral collateral approach to recanalization of a chronically occluded artery.

() 2012, ČKS. Published by Elsevier Urban and Partner Sp. z.o.o. All rights reserved.

\section{Introduction}

Percutaneous coronary intervention $(\mathrm{PCl})$ of chronic total occlusions (CTO) is effective in reducing angina and may also improve left ventricular ejection fraction and long-term survival [1,2]. Despite significant advances in the equipment and technical expertise of the operators, successful recanalization rates of a CTO from an antegrade approach remains suboptimal (60-80\%) [3]. Nevertheless, recent reports have shown success rates of up to $90 \%$ with the retrograde approach $[4,5]$. Currently, the main rationale for choosing the retrograde approach is a previous failed attempt with the conventional antegrade approach, however the inability to delineate the correct ent- rance to the CTO can be dealt with by utilizing the collateral circulation as a primary route instead of the antegrade epicardial artery. In this case report, we describe the successful use of the retrograde approach via an ipsilateral septal collateral from the left anterior descending (LAD) in the treatment of a mid circumflex artery CTO.

\section{Case report}

A 57-year-old man presented with exertional angina (Canadian Cardiovascular Society Angina Class III) of 4-month duration. The chest pain limited his daily activities, including his job that involved heavy physical work. He was an

Address: Vladimír Džavík MD, FSCAI, Interventional Cardiology Program, Peter Munk Cardiac Centre, 6-246 EN, Toronto General Hospital, 
active smoker (1 pack/day), but had no history of hyperlipidemia, hypertension, diabetes mellitus or a family history of coronary artery disease. Initial investigation revealed a normal echocardiogram and inducible ischemia in the basal inferior wall demonstrated on a myocardial perfusion stress test. He was initially given a trial of medical therapy with beta-blockers and nitrates, but did not tolerate the medications, and stated that his symptoms were still interfering with his work and quality of life. Therefore, he was referred to a coronary angiogram that showed a widely patent left main artery, mild irregularities in the LAD and a total occlusion of a dominant circumflex artery beginning just beyond the origin of the first obtuse marginal, that had a chronic appearance. The posterior descending artery and the distal circumflex were filled by mature collaterals from the LAD via the septum. The RCA was non-dominant and had trivial irregularities. The left ventriculogram showed preserved left ventricular systolic function with mild hypokinesis of the apex. Elective $\mathrm{PCl}$ was arranged with plans for the retrograde approach that was considered ideal since the origin of the CTO was not obvious and there was significant septal collateral circulation from the LAD (Fig. 1A). The right radial artery was cannulated with a 7Fr sheath (Cook) and a 7Fr Voda (Boston Scientific) guiding catheter was used to engage the left main. A Pro-Water (Asahi) guidewire was passed antegradely down the LAD and to the third septal perforator. At this point a Corsair microcatheter (Asahi) was loaded on the Pro-Water wire and positioned in the septal branch (Fig. 1B). The Prowater wire was than exchanged for a Fielder FC guidewire (Asahi) that was maneuvered to the posterior descending
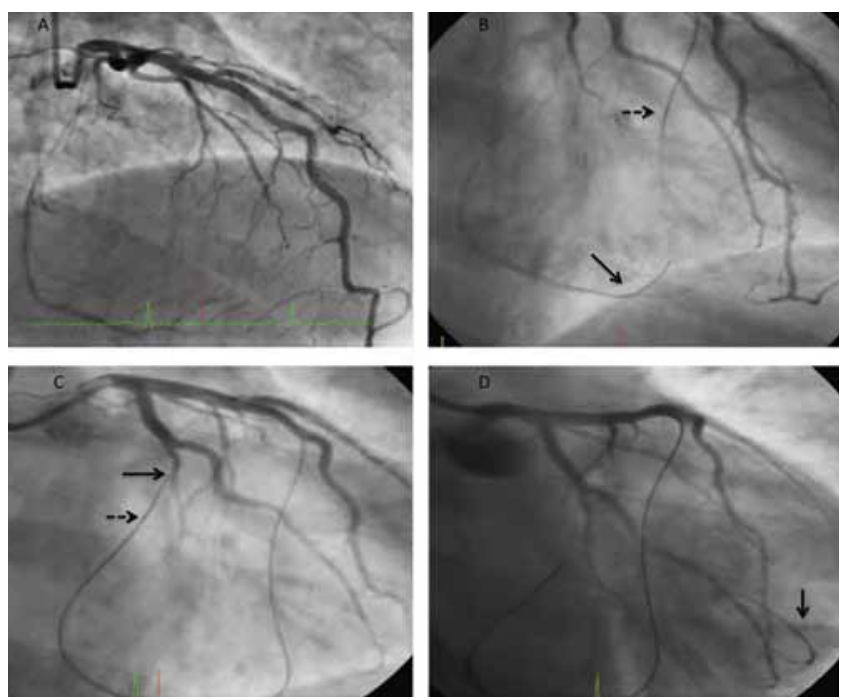

Fig. 1 - (A) Initial angiogram showing a septal retrograde channel and a short occlusion segment, with no entry point visible from the antegrade approach; (B) the tip of a Fielder FC guidewire is visible in the posterior descending artery; the distal tip of the Corsair channel dilator in the septal collateral channel is indicated by the dashed arrow; (C) the Corsair (dashed arrow) is close to the distal entry into the occluded segment, the guidewire positioned to cross the occlusion; (D) a Prowater guidewire is positioned in the large obtuse marginal branch for protection during retrograde crossing of the occlusion. branch and up to the CTO origin (Fig. 1C). At this point a second Pro-Water wire was advanced to protect the first obtuse marginal branch (Fig. 1D). The Fielder FC wire was removed and exchanged for a Miracle $6 \mathrm{mg}$ (Asahi) that was used to cross the CTO (Fig. 2A). The Corsair channel dilating catheter was also removed using an extension wire. A $1.5 \times 15 \mathrm{~mm}$ Voyager balloon (Abbott Vascular) was used to dilate the lesion at 16 atm (Fig. 2B), but the result of this pre-dilation was not enough to attempt crossing the lesion via antegrade approach. Therefore, a $2.0 \times 15 \mathrm{~mm}$ Sprinter Legend balloon (Medtronic) was advanced down the septal branch, but this was not successfully positioned at the CTO due to significant resi-
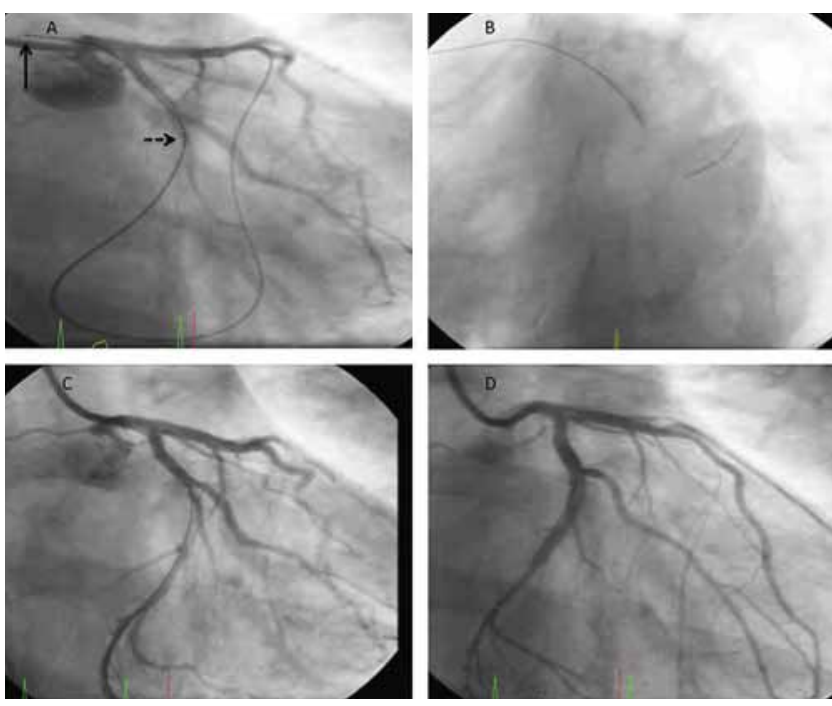

Fig. 2 - (A) The distal tip of a Miracle 6 guidewire is seen in the aortic root (solid arrow), having crossed through the left main ostium; the Corsair tip is indicated by the dashed arrow; (B) a $2.0 \times 15 \mathrm{~mm}$ balloon on the retrograde wire is inflated at the occlusion point after predilation of the septal collateral with a $1.5 \times 15 \mathrm{~mm}$ balloon at 2 atmospheres; (C) a Miracle 3 guidewire is advanced antegradely into the circumflex artery and the retrograde wire, and then the Corsair channel dilator are sequentially removed; (D) the final result following deployment of a Xience Prime LL $3.0 \times 33 \mathrm{~mm}$, and a Xience $V 3.5 \times 12 \mathrm{~mm}$ stent proximally to seal a dissection in the proximal vessel, and removal of the obtuse marginal branch wire.

stance in the septal collateral. We then pre-dilated the septal collateral with a fresh $1.5 \times 15 \mathrm{~mm}$ Voyager balloon (Abbott Vascular) at 2 atm. We were then able to position the $2.0 \times 15 \mathrm{~mm}$ Sprinter Legend balloon (Medtronic) trough the CTO and pre-dilated the lesion at $14 \mathrm{~atm}$ with a good result. A Miracle $3 g$ (Asahi) was then maneuvered antegradely down the circumflex and the Miracle $6 \mathrm{~g}$ wire was removed as the vessel was secured with the antegrade wire. A $2.5 \times 20 \mathrm{~mm}$ Sprinter Legend balloon (Medtronic) at 14 atm was used to pre-dilate the lesion further in order to prepare the vessel for the stent deployment (Fig. 2C). A Xience Prime LL $3.0 \times 33 \mathrm{~mm}$ (Abbott Vascular) was deployed at 14 atm over the lesion. Review angiograms showed a suspicious haziness in proximal edge of the stent. IVUS assessment showed that the angiographic image corresponded to a proximal edge dissection and 
the stent apposition was not optimal in its first $15 \mathrm{~mm}$. A second Xience $3.5 \times 12 \mathrm{~mm}$ (Abbott Vascular) was deployed overlapping with the first stent, sealing the dissection. Stent apposition was optimized using a $4.0 \times 8 \mathrm{~mm}$ Voyager NC balloon (Abbott Vascular). Final angiograms showed an excellent result and TIMI 3 flow (Fig. 2D).

\section{Discussion}

The retrograde approach represents a significant advance in the treatment of chronic total coronary occlusions, since its success rate is significantly higher than the conventional antegrade approach $[4,5]$. However, the necessity of specific expertise and dedicated equipment as well as limited indications still poses a significant challenge to its widespread use by the majority of interventional cardiologists. Currently, there are two main indications for the retrograde approach: a failed antegrade approach and the inability to determine the exact entrance of the CTO. Our patient was referred directly for a CTO intervention with a retrograde approach due to the fact that it was impossible to identify the origin of the CTO using multiple angiographic views.

The treatment of CTOs using the retrograde approach is evolving, however, three routes have been described to reach the distal cap of the occlusion: 1) via a bypass graft in patients who have previously undergone coronary artery bypass graft surgery; 2) via an epicardial collateral; and 3) via a septal perforator. The septal perforator is considered the safest route because it runs within the septal myocardium and can be safely dilated at low pressures with a small calibre balloon to enable delivery of larger balloons in the CTO [6]. Additionally, several strategies for the use of retrograde wires have been introduced such as the controlled antegrade and retrograde tracking (CART) technique, reverse-CART, and true lumen to true lumen entry. The CART and reverse-CART techniques are used to connect the retrograde subintimal channel and antegrade subintimal channel at the site of the CTO $[7,8]$. However, we elected to traverse the occlusion into the true lumen with a dedicated CTO wire and exchange for a different antegrade wire once the CTO was recanalized with a balloon.

To the best of our knowledge, this is the first reported case of retrograde $\mathrm{PCl}$ for a circumflex artery CTO via a septal perforator from the left anterior descending artery. Two prior cases of recanalization of an LAD via ipsilateral septal collaterals have been described, one using a true-to-true technique [9], the other using the CART technique [10]. The use of an ipsilateral collateral presents special challenges. In the case of the use of a contralateral artery, once the occlusion is crossed by the retrograde wire, the procedure is then generally facilitated by externalization of the retrograde wire that then allows for antegrade dilation and stenting of the recanalized vessel. In the case of the ipsilateral approach, this could only be accomplished by bringing a second guide catheter to the ostium of the left main artery [11]. This maneuver could potentially compromise the left main artery. Thus in our view, the most straightforward appro- ach is to advance an adequate length of the retrograde wire, $(5-10 \mathrm{~cm})$ out of the left main ostium into the ascending aorta that will allow for stable position while crossing the septal collateral with balloons. A shorter length out of the left main ostium may result in the wire slipping back distal to the occlusion and thus loss of what has been gained to this point and potential need to recross the occlusion yet again. This approach implies the need for true-to-true lumen crossing of the occlusion. The occlusion must then be dilated retrogradely by adequately sized balloons in order to allow for antegrade crossing by a wire and subsequent antegrade stenting of the recanalized segment. Optimally, the retrograde wire should be removed over the Corsair channel dilating catheter in the septal collateral, once it is confirmed that the antegrade wire is securely positioned in the distal true lumen of the recanalized vessel. Although a 7 French guide catheter was utilized in this case in a patient with a large radial artery, the procedure could also be easily accomplished with a 6 French guide, more suited to the size of radial arteries of the majority of patients [12]. An alternative approach, in the case that the septal collateral cannot be successfully crossed, an unlikely scenario if the Corsair is utilized, may be the use of a second guiding catheter in a "ping-pong" fashion, described by Brilakis et al. in a case of retrograde recanalization of an occluded RCA via an atrial branch, in which case the retrograde wire is externalized into a second guide positioned near the antegrade ostium [11]. Great care must be taken when selecting this approach for the left main artery in order to avoid compromise of this vessel.

\section{Conclusion}

We describe a successful coronary intervention in a circumflex artery CTO using the retrograde approach via septal perforators from the LAD. This report shows that in selected cases the retrograde approach can be successfully performed with a single radial access site and a 7Fr system.

\section{References}

[1] Noguchi T, Miyazaki MS, Morii I, Daikoku S, Goto Y, Nonogi H. Percutaneous transluminal coronary angioplasty of chronic total occlusions. Determinants of primary success and long-term clinical outcome. Catheter Cardiovasc Interv 2000;49:258-64.

[2] Suero JA, Marso SP, Jones PG, Laster SB, Huber KC, Giorgi LV, et al. Procedural outcomes and long-term survival among patients undergoing percutaneous coronary intervention of a chronic total occlusion in native coronary arteries: a 20-year experience. J Am Coll Cardiol 2001;38:409-14.

[3] Thompson CA, Jayne JE, Robb JF, Friedman BJ, Kaplan AV, Hettleman BD, et al. Retrograde techniques and the impact of operator volume on percutaneous intervention for coronary chronic total occlusions an early U.S. experience. JACC Cardiovasc Interv 2009;2:834-42.

[4] Rosenmann D, Meerkin D, Almagor Y. Retrograde dilatation of chronic total occlusions via collateral vessel in three patients. Catheter Cardiovasc Interv 2006;67:250-3. 
[5] Surmely JF, Tsuchikane E, Katoh O, Nishida Y, Nakayama M, Nakamura S, et al. New concept for CTO recanalization using controlled antegrade and retrograde subintimal tracking: the CART technique. J Invasive Cardiol 2006;18: 334-8.

[6] Utsunomiya M, Mukohara N, Hirami R, Nakamura S. Percutaneous coronary intervention for chronic total occlusive lesion of a left anterior descending artery using the retrograde approach via a septal-septal channel. Cardiovasc Revasc Med 2010;11:34-40.

[7] Saito S. Different strategies of retrograde approach in coronary angioplasty for chronic total occlusion. Catheter Cardiovasc Interv 2008;71:8-19.

[8] Joyal D, Thompson CA, Grantham JA, Buller CE, Rinfret $S$. The retrograde technique for recanalization of chronic total occlusions a step-by-step approach. JACC Cardiovasc Interv 2012;5:1-11.
[9] Otsuji S, Terasoma K, Takiuchi S. Retrograde recanalization of a left anterior descending chronic total occlusion via an ipsilateral intraseptal collateral. J Invasive Cardiol 2008;20:312-6.

[10] Suzuki M, Takagi Y, Tsuchikane E. Percutaneous coronary intervention of chronic total occlusion in a left anterior descending coronary artery using an ipsilateral intraseptal bridging collateral tracking technique. Catheter Cardiovasc Interv 2010;76:536-40.

[11] Brilakis ES, Grantham JA, Banerjee S. "Ping-pong" guide catheter technique for retrograde intervention of a chronic total occlusion through an ipsilateral collateral. Catheter Cardiovasc Interv 2011;78:395-9.

[12] Rinfret S, Joyal D, Nguyen CM, Bagur R, Hui W, Leung R, et al. Retrograde recanalization of chronic total occlusions from the transradial approach; early Canadian experience. Catheter Cardiovasc Interv 2011;78:366-74. 